\title{
FRACTURE-DISLOCATION OF THE SHOULDER
}

\section{Report of a Case}

\author{
F. P. Dewar and R. H. Yabsley, Toronto, Canada
}

From the Division of Orthopaedic Surgery, Toronto General Hospital

The results of treatment of fracture-dislocation of the shoulder, in which the greater and lesser tuberosities are fractured and a large capital fragment is dislocated are not usually very good (Neer, Brown and Mclaughlin 1953; Knight and Mayne 1957). The production of this four-fragment fracture, as described by Codman (1934), makes closed reduction hard to achieve and open reduction very difficult.

When this injury occurs in an elderly patient conventional methods of management may be sufficient to provide restoration of some movement. But when it occurs in a young person who may need to do heavy work for many years to earn his living, existing methods of treatment often fall short in providing him with a shoulder which is comfortable, mobile and strong. Excision of the humeral head often results in a joint that is painful and weak (Watson-Jones 1955, Knight and Mayne 1957). Neer et al. (1953) studied twenty patients, nineteen of whom had had excision of the humeral head with or without reconstruction. The usual range of scapulo-humeral movement was only 5-25 degrees, and five of nineteen patients developed a solid bony bridge across the joint.

Many authors advise against excising the humeral head, stating that even if severe avascular necrosis occurs, the results are better than if the head has been excised (Codman 1934, Watson-Jones 1955, Neviaser 1962).

In a group of sixteen patients who had suffered fracture-dislocation of the shoulder, characterised by a single capital fragment containing most of the articular surface of the humeral head which was replaced by operation, Knight and Mayne (1957) observed that thirteen showed avascular necrosis of the humeral head.

As the results obtained by these methods in young persons are poor, and as it is inadvisable to use a prosthesis at this time of life, we invented the following operation based upon a principle described by Moore (1948) for the treatment of ununited fractures of the femoral neck.

\section{CASE REPORT}

A journalist of thirty-two was helping a friend to lift a heavy table up a staircase when the table slipped. It pinned the patient against the wall, injuring his left shoulder. Radiological examination several hours later showed an anterior fracture-dislocation of the left shoulder of the four-fragment variety. Most of the articular surface of the joint seemed to be contained on the large capital fragment (Fig. 1). There was no involvement of the neurovascular bundle. A short while later the fracture-dislocation was exposed through a delto-pectoral incision. The acromion was divided and the capsule of the joint was opened. The head was found to be free of all soft-tissue attachments and was removed from the wound. Little damage had happened to the articular surface.

After the technique of Moore (1948) a Smith-Petersen reamer and a burr were used to remove the bulk of the avascular bone in the fragment, leaving only a thin subchondral layer of cortical bone. The fractured end of the humeral shaft was then shaped and smoothed to 
receive the osteochondral implant. This was placed with the required amount of retroversion and inclination, and was held in place with a screw through the "cup." The screw was countersunk beneath the articular surface and passed downwards to the outer cortex of the humeral shaft.

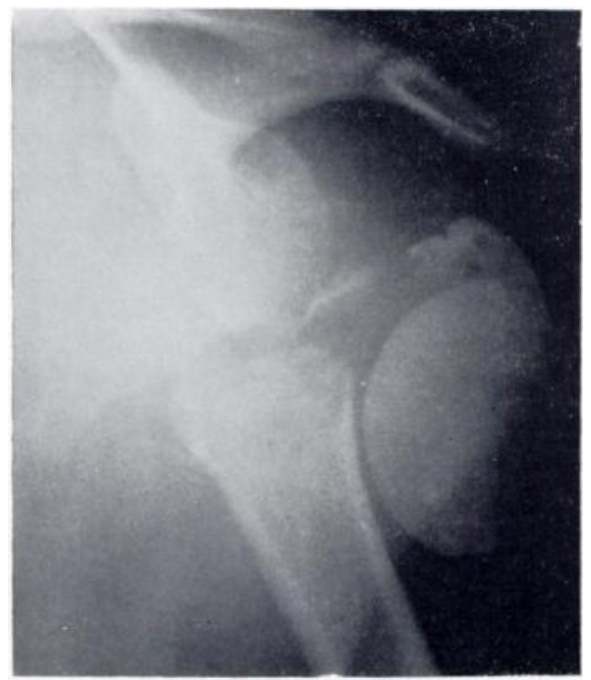

FIG. 1

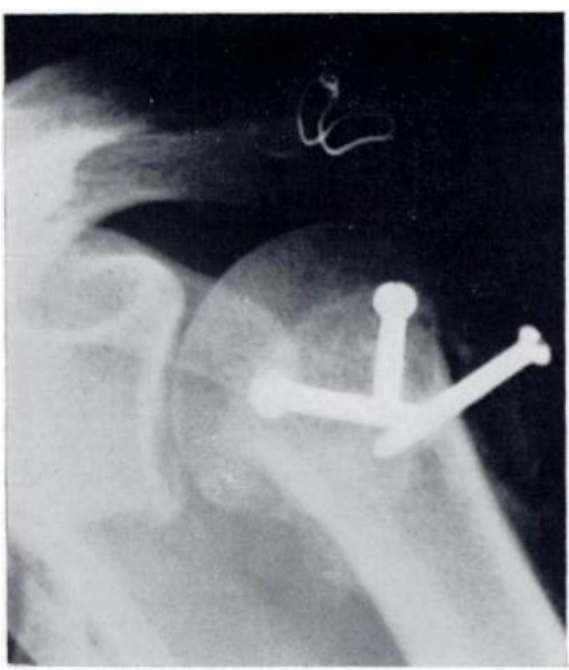

FIG. 2

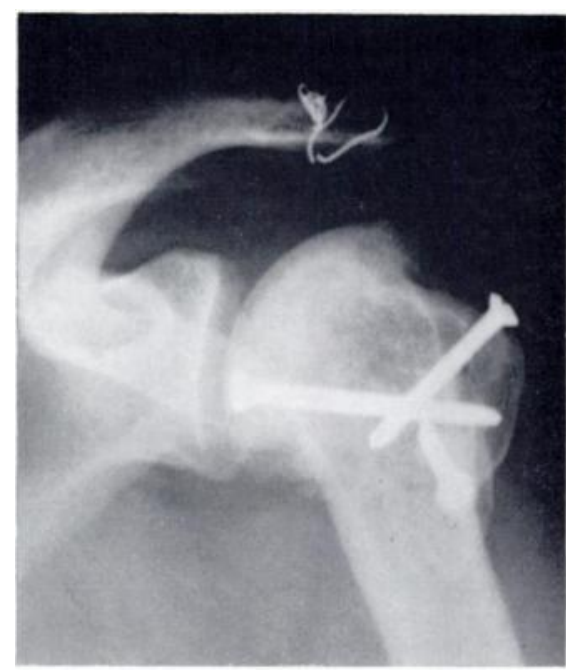

FIG. 3

Figure 1-The fracture-dislocation before operation. Figure 2-Radiograph of the shoulder after operation. The capital fragment and the greater and lesser tuberosities are held with screws and the acromion has been replaced. The capital fragment has been reamed out. Figure 3Radiograph of the shoulder five and a half years after operation. There is no evidence of avascular necrosis and the humeral fragment has remained smooth.

The greater tuberosity containing the intact insertion of the supraspinatus tendon was reattached to the lateral humeral shaft a little lower than normal with a single screw. The lesser tuberosity which had been detached by the subscapularis tendon was similarly reattached with another screw (Fig. 2). The wound was closed and the arm was supported in a Velpeau bandage. The patient was encouraged to begin active movements of the shoulder as soon as it was comfortable.

VOL. 49 B, NO. 3, AUGUST 1967 
When examined five and a half years after injury he had an excellent range of movement at the shoulder (Fig. 4). He stated that he was able to carry out his duties as a journalist and that he had only mild intermittent discomfort in the shoulder. He felt that he had regained 90 per cent of his former strength. Radiographs showed an excellent joint space, and no evidence of avascular necrosis (Fig. 3).

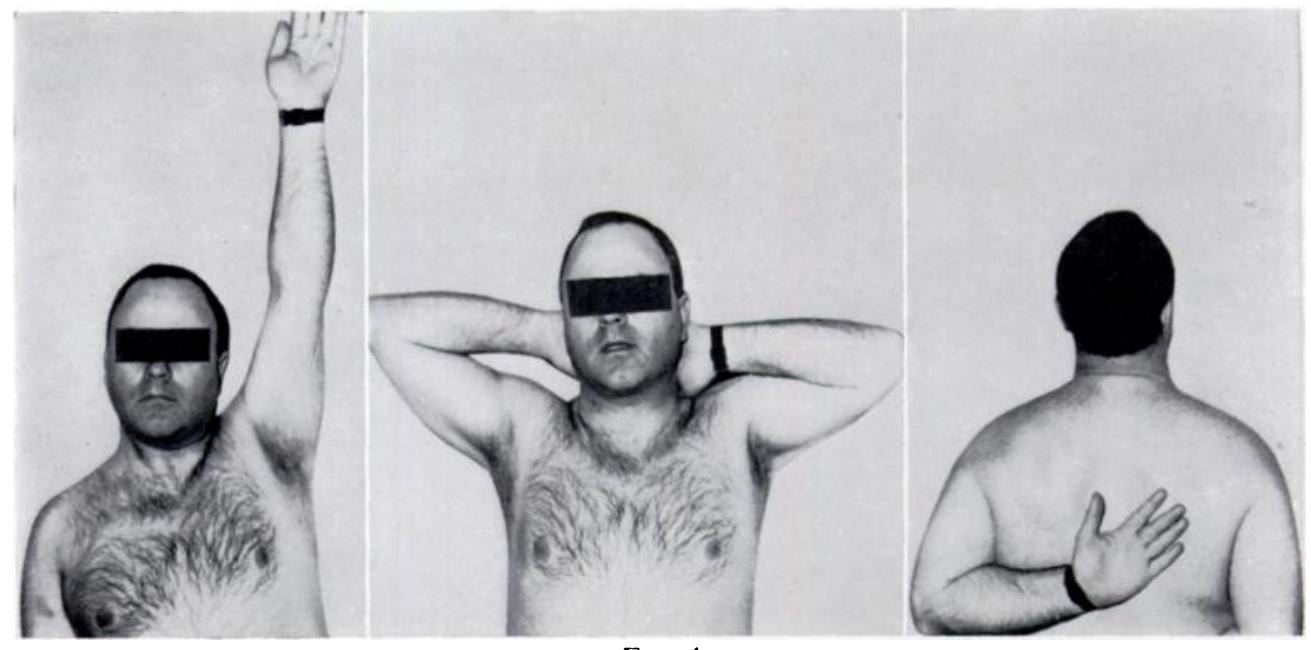

FIG. 4

Range of shoulder movement five and a half years after operation.

\section{DISCUSSION}

This procedure was carried out in a young adult. Moore (1948), reviewing eleven patients in whom the same procedure was done for ununited fractures of the femoral neck, reported nine excellent results. DePalma. Tsaltas and Mauler (1963) studied the fate of femoral heads in dogs, in which the head was removed and replaced after curettage of the cancellous bone leaving a thin layer of bone beneath the articular cartilage. They observed that the osseous portion of the graft was completely replaced by the thirty-fourth day, and that there had been survival of the articular cartilage. They believed that the stimulus provided by physiological function played an important role in the ultimate fate of the graft. Support for this theory was provided by Pap and Krompecher (1961) who demonstrated the importance of physiological stimulus in the survival of transplanted articular cartilage in the knee.

Merle d’Aubigné. Postel. Mazabraud. Massias and Gueguen (1965) showed that the articular cartilage in idiopathic avascular necrosis of the femoral head survived until revascularisation advanced to the subchondral region. Softening and collapse then occurred. leading to removal of the firm base in the articular cartilage which then degenerated and died. As the thin shell of bonè beneath the articular cap in our procedure was rapidly revascularised a firm base was maintained for the articular cartilage.

Ekholm (1955). using radioactive gold injection studies and autoradiography, showed that articular cartilage. in addition to being nourished by synovial fluid, is also nourished to some extent from the medullary route.

We suggest that this procedure ensures viability of the cartilage by providing clear passage of nutriment through both routes.

\section{SUMMARY}

The case is reported of a fracture-dislocation of the shoulder, treated by an operation based upon a principle advanced by Moore in 1948. An excellent radiological and functional result was observed five and a half years after the injury. 


\section{REFERENCES}

Codman, E. A. (1934): The Shoulder, pp. 285 and 314. Boston: Thomas Todd Company (Printers).

DePalma, A. F., Tsaltas, T. T., and Mauler, G. G. (1963): Viability of Osteochondral Grafts as Determined by Uptake of $\mathrm{S}^{35}$. Journal of Bone and Joint Surgery, 45-A, 1565.

Екноцм, R. (1955): Nutrition of Articular Cartilage: A Radioautographic Study. Acta Anatomica, $24,329$.

KNight, R. A., and Mayne, J. A. (1957): Comminuted Fractures and Fracture-dislocations Involving the Articular Surface of the Humeral Head. Journal of Bone and Joint Surgery, 39-A, 1343.

Merle D'Aubigné, R., Postel, M., Mazabraud, A., Massias, P., and Gueguen, J. (1965): Idiopathic Necrosis of the Femoral Head in Adults. Journal of Bone and Joint Surgery, 47-B, 612.

MOORE, J. R. (1948): Cartilaginous-cup Arthroplasty in Ununited Fractures of the Neck of the Femur. Journal of Bone and Joint Surgery, 30-A, 313.

Neer, C. S., Brown, T. H., Jun., and Mclaughlin, H. L. (1953): Fracture of the Neck of the Humerus with Dislocation of the Head Fragment. American Journal of Surgery, 85, 252.

Neviaser, J. S. (1962): Complicated Fractures and Dislocations about the Shoulder Joint. Journal of Bone and Joint Surgery, 44-A, 984.

PAP, K., and Krompecher, S. (1961): Arthroplasty of the Knee: Experimental and Clinical Experiences. Journal of Bone and Joint Surgery, 43-A, 523.

Watson-Jones, Sir R. (1955): Fractures and Joint Injuries. Fourth edition, p. 501. Edinburgh and London: E. \& S. Livingstone Ltd. 